HUB-EP-99/56

LPTENS-99/35

\title{
Continuous Gauge and Supersymmetry Breaking for Open Strings on D-branes
}

\author{
Ralph Blumenhagen ${ }^{1}$, Costas Kounnas ${ }^{2,3}$ and Dieter Lüst ${ }^{4}$ \\ 1,4 Humboldt-Universität Berlin, Institut für Physik, Invalidenstrasse 110, \\ 10115 Berlin, Germany \\ 2 Laboratoire de Physique Théorique, ENS, F-75231 Paris, France \\ 3 Theory Divison, CERN, CH-1211, Geneva 23, Switzerland
}

\begin{abstract}
We consider freely acting orbifold compactifications, which interpolate in two possible decompactification limits between the supersymmetric type II string and the nonsupersymmetric type 0 string. In particular we discuss how D-branes are incorporated into these orbifold models. Investigating the open string spectrum on D3-branes, we will show that one can interpolate in this way between $\mathcal{N}=4$ supersymmetric $U(N)$ respectively $U(2 N)$ Yang-Mills theories and non-supersymmetric $U(N) \times U(N)$ gauge theories with adjoint massless scalar fields plus bifundamental massless fermions in a smooth way. Finally, by lifting the orbifold construction to M-theory, we conjecture some duality relations and show that in particular a new supersymmetric branch of gauge like theories emanate for the non-supersymmetric model.
\end{abstract}

$10 / 99$

${ }^{1}$ e-mail: blumenha@physik.hu-berlin.de

3 e-mail: costas.kounnas@cern.ch

4 e-mail: luest@physik.hu-berlin.de 


\section{Introduction}

As it became clear during the recent years, the emergence of $(p+1)$-dimensional, supersymmetric gauge theories from open strings living on the world volumes of Dp-branes in closed string theories provides very important insights into the perturbative and nonperturbative gauge theory dynamics. Of particular interest is the limit in which the closed (bulk) string degrees of freedom decouple from the open (boundary) degrees of freedom; this limit is provided on the $U(N)$ gauge theory side by taking the number of color degrees of freedom to be very large, $N \rightarrow \infty$, whereas on the closed string side the same limit is achieved by considering the classical weak coupling limit, $g_{s} \rightarrow 0$. Quite surprisingly, there is in fact a very striking duality between the open string gauge theories on the boundary and the closed string gravitational theories in the bulk, stating that superconformal gauge theories in the large $\mathrm{N}$ limit are dual to type II superstring theories in anti-de-Sitter background spaces [1 [1] The most well understood example is given by the duality between $\mathcal{N}=4, U(N)$ Yang-Mills gauge theory and supergravity in an $A d S_{5} \times S^{5}$ background.

Of course the most interesting problem is to explore the CFT/AdS duality for models which allow for (spontaneous) breaking of space-time supersymmetry. One way to break supersymmetry in four dimensions is to consider models with non-zero temperature [4]. A different and perhaps more direct way to construct non-supersymmetric string models is provided by the type 0 string construction [5-7] following an idea by Polyakov [8]. Seen from a world sheet point a view the non-supersymmetric type 0A/B theories are simply obtained by a non-supersymmetric, but nevertheless modular invariant GSO projection [9,10], such that the spectrum of closed strings is purely bosonic. Alternatively the type 0 strings can be constructed as an orbifold of type II superstring theory [11] by modding out the symmetry $(-1)^{F_{s}}$, where $F_{s}$ denotes the space-time fermion number. In this way all closed string fermions in the (R,NS) and (NS,R) are projected out by the $\mathbb{Z}_{2}$ action, whereas the twisted sector contains an (NS,NS) tachyon and in addition a second set of massless $(\mathrm{R}, \mathrm{R})$ fields. This has the important effect that the D-branes present in type 0 theories are essentially doubled compared to the supersymmetric type II parent theories. We will call the two different kinds of p-dimensional D-branes (electric) Dp and (magnetic) Dp'-branes. Implementing the $(-1)^{F_{s}}$ projection in the open string sector, it turns out that the open strings stretched between D-branes of the same kind, i.e. between Dp and Dpbranes respectively between Dp' and Dp'-branes, lead to space-time bosons, in particular to massless gauge bosons and massless scalar fields. On the other hand, open strings 
between Dp and Dp'-branes lead to space-time fermions. Let us mention that recently also non-supersymmetric tachyon-free compactifications of both type II string theory [12-16] and type 0 string theory [17-23] were discussed.

Using this construction an interesting class of non-supersymmetric gauge models arises when considering an equal number of $N$ electric D3 and $N$ magnetic D3'-branes in type 0B string theory. This configuration leads to a non-supersymmetric $U(N)_{e} \times U(N)_{m}$ gauge theory with bosonic and fermionic massless matter arranged in such a way that the $\beta$-function vanishes to leading order in $1 / N$. In fact, Klebanov and Tseytlin [5-7] gave reasonable evidence that this gauge theory is again dual to a tachyon-free gravity background of the form $A d S_{5} \times S^{5}$ just as in the supersymmetric type IIB theory. Putting the type 0B D3-branes on transversal singularities [24-27] or considering type 0A HananyWitten [28] like brane constructions a full variety of non-supersymmetric gauge theories $\left(U(N)_{e} \times U(N)_{m}\right)^{K}$ can be constructed [29-33], where $K$ is a number which characterizes the singularity type or the number of NS 5-branes in the Hanany-Witten set up. All these models are Bose-Fermi degenerated and have the same one-loop $\beta$-functions as their corresponding $U(N)^{K}$ type II parent models. This correspondence becomes even more close by noting that the gauge theory given by the diagonal $U(N)_{e+m} \in U(N)_{e} \times U(N)_{m}$ is identical to the gauge theory of the underlying type II theory. Therefore one might conjecture that there exists a smooth interpolation between the supersymmetric $U(N)$ gauge theories and the non-supersymmetric $U(N) \times U(N)$ models in such a way that supersymmetry is smoothly broken (installed) along the line of deformation. Since the $U(N)_{e} \times U(N)_{m}$ gauge theories do not contain any massless scalars fields with the right quantum numbers to trigger the symmetry breaking to the diagonal group $U(N)_{e+m}$ via the usual field theoretical Higgs mechanism, one has to consider the couplings between the open string boundary modes to the closed string bulk modes in order to realize the desired interpolation.

One well established way to break supersymmetry in a smooth way is provided by the Scherk-Schwarz mechanism [34-39]. This class of models is built as freely acting orbifolds with different boundary conditions for bosons and fermions, very similar to strings at finite temperature 40 45]. Sending the radius $R$ of the orbifold to infinity (to zero) spacetime supersymmetry is recovered. Recently Scherk-Schwarz supersymmetry breaking was studied in type I compactifications [4649] and also in M-theory [50]. Moreover the ScherkSchwarz mechanism was also used to show that there exist indeed two types of models which smoothly interpolate between the closed type IIA/B and type 0A/B theories [51]. 
They can be either realized as a freely acting orbifold of type II on $S^{1} /(-1)^{F_{s}} S$, where $S$ denotes a half shift on the circle, or alternatively as a freely acting orbifold of type 0 on $S^{1} /(-1)^{f_{L}} S$, where $f_{L}$ is the left-moving world sheet fermion number. In the former case supersymmetry is recovered for infinite radius of the circle, whereas in the latter case the zero radius limit is fully supersymmetric. These interpolating models are expected to exhibit a Hagedorn phase transition at the special critical radius of the circle where a tachyonic mode arises [42,45]. The freely acting orbifold of type IIA can also be lifted to M-theory [51]. In this case it was postulated in [51] that the strong coupling regime of type $0 \mathrm{~A}$ is given by M-theory on $S^{1} /(-1)^{F_{s}} S$. Taking this conjecture seriously, one implication is that at strong coupling the type $0 \mathrm{~A}$ string becomes supersymmetric in the bulk. Moreover, the closed string tachyon becomes massive at strong coupling and at the same time, fermions, which are of solitonic nature in the type 0A string, become light and provide the massless (R-NS) fermions of the closed type IIA superstring.

In this paper we will extend this construction including also the D-branes and the corresponding open strings into the orbifolds which interpolate between type II and type 0 strings. In this way we will show that one can smoothly interpolate between broken and unbroken gauge theories and at the same time between supersymmetric and nonsupersymmetric models in a way not known before from field theory. We will first consider type IIA/B compactified on $S^{1} /(-1)^{F_{s}} S$ where $N$ Dp-branes (p even (odd) for type A(B)) are either wrapped around the orbifold circle, or are placed transversal to it. In the former case one interpolates between a $(p+1)$-dimensional, $\mathcal{N}=4$ supersymmetric $U(2 N)$ gauge theory at infinite radius and a $p$-dimensional, non-supersymmetric $U(N) \times U(N)$ gauge at zero radius. In case of transversal branes, the infinite radius limit of the orbifold provides an $\mathcal{N}=4$ supersymmetric $U(N)$ gauge theory in $p+1$ dimensions whereas in the zero radius limit a non-supersymmetric $U(N) \times U(N)$ gauge theory in $p+2$ dimensions appears. We extend this construction by considering two-dimensional type IIA/B orbifold compactifications on $S^{1} \otimes S^{1} /(-1)^{F_{s}} S$. In turns out that varying the two radii of the compact space one can interpolate between supersymmetric and non-supersymmetric gauge theories living in the same space-time dimension. A similar, in fact T-dual, picture arises from D-branes of type 0A/B on $S^{1} /(-1)^{f_{L}} S$ and on $S^{1} \otimes S^{1} /(-1)^{f_{L}} S$, respectively.

Finally we will discuss how this construction can be embedded into M-theory. Here again the M-theory moduli allow to interpolate between weakly coupled nonsupersymmetric gauge theories and strongly coupled supersymmetric gauge theories in 
a smooth way. In contrast to the string theory embeddings, here one of the radii is related to the gauge coupling constant, so that one is really interpolating between small and strong gauge coupling. Taking the non-supersymmetric dualities seriously, we are led to strong-weak coupling dualities for the non-supersymmetric gauge theories. Moreover, we see that non-supersymmetric gauge theories contain new branches at strong coupling, which are supersymmetric, but not all stringy degrees of freedom are decoupled.

\section{Type II compactifications on freely acting orbifolds}

\subsection{Supersymmetry restoration in the bulk}

In this section we briefly review the smooth orbifold construction of [51] which produces type 0 from type II and vice versa. One starts with the type IIA (B) superstring compactified in the ninth direction on a circle $S^{1}$ of radius $R_{9}^{I I}$. The theory contains 32 unbroken supercharges for every value of $R_{9}^{I I}$. In the limit $R_{9}^{I I} \rightarrow \infty$ the Kalazu-Klein modes become massless and one recovers the type IIA (B) superstring in ten dimensions. On the other hand, for $R_{9}^{I I} \rightarrow 0$ the winding modes become massless and the theory is now identical to the type IIB (A) superstring in ten dimensions. This is what is usually called T-duality between the type IIA/B superstring [52,53] (for a recent discussion on IIA/IIB T-duality and M-theory see [54]).

At the next step we build the orbifold type IIA (B) on $S^{1} /(-1)^{F_{s}}$ of radius $R_{9}^{0}$. This $\mathbb{Z}_{2}$ projection breaks all 32 supercharges and the resulting theory is nothing else than the type 0 string compactified on $S^{1}$ with purely bosonic spectrum in the closed string bulk. For large $R_{9}^{0} \rightarrow \infty$ the type 0A (B) string in ten dimensions emerges whereas for $R_{9}^{0} \rightarrow 0$ the ten-dimensional type 0B (A) string is present. Hence $T$-duality among type 0A/B just works like for the type IIA/B superstring pair.

Finally we combine the action of the $\mathbb{Z}_{2}(-1)^{F_{s}}$ orbifold projection with the half-shift $S$ along the circle, i.e. we are now considering type IIA (B) on the freely acting orbifold $S^{1} /(-1)^{F_{s}} S$ of radius $R_{9}$. For finite values of $R_{9}$ all 32 supercharges are broken in the sense that all 32 gravitinos are massive with mass of order $1 / R_{9}$. In the limit $R_{9} \rightarrow \infty$ the gravitinos become massless, and one obtains the closed string spectrum of the tendimensional type IIA (B) superstring. On the other hand, for $R_{9} \rightarrow 0$ the gravitinos become infinitely heavy such that they completely decouple, and one regains the spectrum of the type $0 \mathrm{~A}(\mathrm{~B})$ for $R_{9}^{0}=0$. So effectively, using also the type 0 T-duality, the limit $R_{9} \rightarrow 0$ of type IIA (B) on this orbifold is given by the ten-dimensional type 0B (A) string 
theory. There is a tachyon for $R_{9}<\sqrt{2}$, which decouples in the limit $R_{9} \rightarrow \infty$. Therefore one expects a Hagedorn phase transition at this radius [43-45]. Note that at infinite radius the two moduli spaces of type II compactified on the circle $S^{1}$ and of type II compactified on the orbifold $S^{1} /(-1)^{F_{s}} S$ meet. In the same way, at $R_{9}=0$ the moduli space of type 0 on $S^{1}$ and of type II on $S^{1} /(-1)^{F_{s}} S$ have a common intersection.

\subsection{D-branes and open strings in Type II on $S^{1} /(-1)^{F_{S}} S$}

First let us briefly recall the D-brane spectrum of type 0 strings. As already mentioned, the $(-1)^{F_{s}}$ projection removes all closed string fermions from the untwisted sector and leads to a tachyon and further massless RR fields in the twisted sector. Computing the spectrum one finds that all states in the RR sector are doubled implying that all Dp-branes (p odd in type 0B, p even in type 0A) are doubled, as well. In particular, in the case of D3branes there now exist (electric) D3 and (magnetic) D3'-branes. Using the boundary state approach it was shown in [19] that indeed the boundary state representing a Dp-brane in type II splits into two boundary states of type 0. The explicit form of the boundary states was used to derive rules for open strings stretched between the various types of Dp-branes. Open strings stretched between the same kind of Dp-branes carry only spacetime bosonic modes, whereas open strings stretched between a Dp and a D $p^{\prime}$-brane carry only space-time fermionic modes. The massless spectrum living on the D3-branes is given by a four-dimensional gauge theory with gauge group $G=U(N) \times U(N)$, three complex bosons in the adjoint and four Weyl-fermions in the $(\mathrm{N}, \overline{\mathrm{N}})+(\overline{\mathrm{N}}, \mathrm{N})$ representation of $G$. It follows that the one-loop $\beta$-function vanishes, and as was shown explicitly in [6] the two-loop $\beta$-function vanishes in the large $\mathrm{N}$ limit. In the next to leading order one obtains a non-vanishing contribution to the two-loop $\beta$-function coefficient, $b_{2}=-16$. Thus, the gauge theory is free in the infrared.

Next consider the type II string compactified on $S^{1} /(-1)^{F_{s}}$. First we discuss the case where the circle $S^{1}$ is transversal to the Dp and Dp'-branes such that the D-branes take certain positions on $S^{1}$. In addition to the open strings discussed before there are also winding open strings which are wrapped $n$-times around $S^{1}$ before their ends are stuck at the D-branes. Therefore we call this case the winding picture. The masses of these winding states are proportional to $\left|n R_{9}^{0}\right|$. On the one hand, for $R_{9}^{0} \rightarrow \infty$ the winding modes become heavy and decouple. On the other hand for $R_{9}^{0} \rightarrow 0$ an infinite number of winding modes becomes massless, which means that effectively the world volume dimension of a Dp-brane grows by one unit. 
Alternatively we can also decide to put the compact circle along one of the world volume directions of the Dp-branes. This is called the momentum picture, since there are open strings on the $\mathrm{Dp}\left(\mathrm{p}\right.$ ')-branes which carry discrete momenta of the order $m / R_{9}^{0}$. Hence these states become light in the limit $R_{9}^{0} \rightarrow \infty$, but decouple in the other decompactification limit $R_{9}^{0} \rightarrow 0$. Thus, in the $R_{9}^{0} \rightarrow \infty$ limit the dimension of the brane world-volume is effectively enlarged.

After these considerations we are now ready to discuss the D-branes and open strings within the compactification of type II string on the orbifold $S^{1} /(-1)^{F_{s}} S$.

\section{(i) The winding picture: transversal Dp-branes}

For concreteness let us consider the type IIB string compactified on a circle of radius $R_{9}$. We place $2 \mathrm{~N}$ transversal D3-branes in this background and divide by the $\mathbb{Z}_{2}$ operation $T=(-1)^{F_{s}} S$. The shift acts on a momentum/winding ground state as $S|m, n\rangle=(-1)^{m}|m, n\rangle$. Let us first consider the range $0<R_{9}<\infty$ and then consider the two possible limits. Requiring that $T$ is indeed a symmetry of the background, we have to make sure that the D3-branes are arranged in such a way that $T$ transforms one brane into another. We will place $\mathrm{N}$ branes at a position $x_{9}=A=0$ on the circle and $\mathrm{N}$ branes at $x_{9}=B=R_{9} / 2$. Therefore we deal with two kinds of winding strings in the open string sector. First there are the AA- and BB-sectors with open strings between two D3-branes either at $x_{9}=0$ or at $x_{9}=R_{9} / 2$ and integer winding numbers $w=n R_{9}$. Their masses are given by

$$
M^{2} \sim\left(n R_{9}\right)^{2} .
$$

Second in the AB- and BA-sectors there are open strings stretched between one D3-brane at $x_{9}=0$ and one other D3-brane at $x_{9}=R_{9} / 2$. These states have half-inter winding numbers $w=\left(n+\frac{1}{2}\right) R_{9}$, and their masses are given by

$$
M^{2} \sim\left(\left(n+\frac{1}{2}\right) R_{9}\right)^{2} .
$$

Hence the ground state in the $\mathrm{AB}(\mathrm{BA})$-sector is massive for finite $R_{9}$.

The natural action of $T$ on the Chan-Paton factors of these branes is given by

$$
\gamma_{T}=\left(\begin{array}{ll}
0 & I \\
I & 0
\end{array}\right)_{2 N, 2 N}
$$


so that the branes at $x_{9}=0$ are mapped to the branes at $x_{9}=R_{9} / 2$. Computing the annulus amplitude for open string stretched between two D3-branes is straightforward and gives

$$
\begin{aligned}
A & =\operatorname{Tr}\left[\frac{1+T}{2} P_{G S O} e^{-2 \pi t L_{0}}\right] \\
& =\frac{N^{2}}{2} \frac{\vartheta\left[\begin{array}{l}
0 \\
0
\end{array}\right]^{4}-\vartheta\left[\begin{array}{c}
0 \\
1 / 2
\end{array}\right]^{4}-\vartheta\left[\begin{array}{c}
1 / 2 \\
0
\end{array}\right]^{4}}{\eta^{12}} \sum_{n \in \mathbb{Z}}\left(e^{-2 \pi t R_{9}^{2} n^{2}}+e^{-2 \pi t R_{9}^{2}\left(n+\frac{1}{2}\right)^{2}}\right)
\end{aligned}
$$

with argument $e^{-2 \pi t}$. The first term with integer windings is from open strings stretched between branes at the same location and the second term with half-integer windings is from open strings stretched between branes at opposite locations of the circle. It is straightforward to compute the massless spectrum. In the range $R_{9}>0$ we obtain the four dimensional supersymmetric spectrum shown in Table 1.

\begin{tabular}{|l|l|l|}
\hline sector & spin & gauge $U(N)$ \\
\hline AA, BB & vector & Adj \\
& scalar & $6 \times \mathbf{A d j}$ \\
& Weyl & $4 \times \mathbf{A d j}$ \\
\hline
\end{tabular}

Table 1: $\quad$ Winding picture: open string spectrum of $S^{1} / T$ for finite $R_{9}$

Thus the massless spectrum in the AA and BB sectors is exactly the one of $U(N) \mathcal{N}=4$ super Yang-Mills theory in four space-time dimensions. In the limit $R_{9} \rightarrow \infty$ the mass of the other winding states becomes infinite and they decouple. Therefore we are dealing with four-dimensional $U(N) \mathcal{N}=4$ supersymmetric Yang-Mills in this decompactification limit.

In the $R_{9} \rightarrow 0$ limit however an infinite number of winding states in the AA and $\mathrm{BB}$ sectors becomes massless. This has the effect that the gauge theory now lives in one dimension higher. Moreover the gauge group gets enlarged, as now open strings in the AB and BA sectors stretched between the D3-branes at locations A and B become massless. By a unitary transformation

$$
U=\frac{1}{\sqrt{2}}\left(\begin{array}{cc}
I & I \\
I & -I
\end{array}\right)_{2 N, 2 N}
$$


one obtains $\gamma_{T}=\operatorname{diag}[I,-I]$ and now one is in the situation of the pure $(-1)^{F_{s}}$ orbifold, which leads to the massless open string spectrum listed in Table 2.

\begin{tabular}{|l|l|l|}
\hline sector & spin & gauge $U(N) \times U(N)$ \\
\hline $33,3^{\prime} 3^{\prime}$ & vector & $(\mathbf{A d j}, 1)+(1, \mathbf{A d j})$ \\
& scalar & $5 \times\{(\mathbf{A d j}, 1)+(1, \mathbf{A d j})\}$ \\
\hline $33^{\prime}, 3^{\prime} 3$ & Dirac & $2 \times\{(N, \bar{N})+(\bar{N}, N)\}$ \\
\hline
\end{tabular}

Table 2: $\quad$ Winding picture: open string spectrum of $S^{1} / T$ for $R_{9}=0$

This is precisely the mass spectrum of type 0B with $N$ electric D3 plus $N$ magnetic D3'-branes but at zero radius $R_{9}^{0 B}=0$. This means that the non-supersymmetric gauge theory actually lives in five space-time dimensions, i.e. this limit is nothing else than the type 0A string with a non-supersymmetric $U(N) \times U(N)$ gauge theory arising from $N$ electric D4 plus $N$ magnetic D4'-branes.

In summary, so far we have constructed an interpolating model between fourdimensional $\mathcal{N}=4$ super YM theory at $R_{9} \rightarrow \infty$ and a special five-dimensional nonsupersymmetric gauge theory at $R_{9} \rightarrow 0$. The additional massless states appear in the winding sector of open strings; hence there is no field theoretic description for them. $R_{9}$ is a purely stringy parameter, which is not present in conventional gauge theory. The corresponding closed string modulus field in the bulk is coupled to the open strings in the boundary and provides at the same time the supersymmetry restoration and the gauge symmetry breaking on the D-branes.

Of course, this picture can be immediately generalized considering $2 \mathrm{~N}$ Dp-branes, $\mathrm{p}$ even (odd), in type IIA(B) superstring compactified on $S^{1} /(-1)^{F_{s}} S$, where again half of the Dp-branes are positioned at $x_{9}=0$ and the other half sit at $x_{0}=R_{9} / 2$. In this way one interpolates between supersymmetric type IIA(B) Dp-branes at $R_{9} \rightarrow \infty$ and non-supersymmetric type $0 \mathrm{~B}(\mathrm{~A}) \mathrm{D}(\mathrm{p}+1)-, \mathrm{D}(\mathrm{p}+1)^{\prime}$-branes at $R_{9} \rightarrow 0$. In the open string sector one is smoothly interpolating between $(p+1)$-dimensional supersymmetric $U(N)$ Yang-Mills theory with 16 supercharges at $R_{9}=\infty$ and $(\mathrm{p}+2)$-dimensional nonsupersymmetric $U(N) \times U(N)$ Yang-Mills theory at $R_{9}=0$. 
(ii) The momentum picture: wrapped Dp-branes

Now let us discuss the case where the orbifold $S^{1} /(-1)^{F_{s}} S$ lies in one of the world volume directions of the D-branes, i.e. the D-branes are wrapped around the compact orbifold. The open string states again split into two sectors, namely momentum states with even momenta $p_{9}=2 \mathrm{~m} / R_{9}$ and masses

$$
M^{2} \sim(2 m)^{2} / R_{9}^{2}
$$

and in general different states with odd momenta $p_{9}=(2 m+1) / R_{9}$ and corresponding masses

$$
M^{2} \sim(2 m+1)^{2} / R_{9}^{2}
$$

To be specific consider 2N D4-branes of type IIA wrapped in this way. For finite values of $R_{9}$ the resulting gauge theory lives in four uncompactified space-time dimensions, whereas in the $R_{9} \rightarrow \infty$ limit momentum modes are massless, and the gauge theory become fivedimensional. Since in the $R_{9} \rightarrow 0$ limit we would like to obtain the non-supersymmetric gauge theory with gauge group $U(N) \times U(N)$ we make the following choice for the action of $T$ on the Chan-Paton labels

$$
\gamma_{T}=\left(\begin{array}{cc}
I & 0 \\
0 & -I
\end{array}\right)_{2 N, 2 N}
$$

In order to obtain the precise form of the spectrum we compute the annulus diagram for open strings stretched between the wrapped D4-branes.

$$
A=\operatorname{Tr}\left[\frac{1+T}{2} P_{G S O} e^{-2 \pi t L_{0}}\right]=N^{2} \frac{\vartheta\left[\begin{array}{l}
0 \\
0
\end{array}\right]^{4}-\vartheta\left[\begin{array}{c}
0 \\
1 / 2
\end{array}\right]^{4}-\vartheta\left[\begin{array}{c}
1 / 2 \\
0
\end{array}\right]^{4}}{\eta^{12}} \sum_{m \in \mathbb{Z}}\left(e^{-2 \pi t \frac{m^{2}}{R_{9}^{2}}}\right)
$$

The resulting massless spectrum for $R_{9}<\infty$ is listed in Table 3 .

\begin{tabular}{|l|l|l|}
\hline sector & spin & gauge $U(N) \times U(N)$ \\
\hline \multirow{3}{*}{$p_{9}$ even } & vector & $(\mathbf{A d j}, 1)+(1, \mathbf{A d j})$ \\
& scalar & $6 \times\{(\mathbf{A d j}, 1)+(1, \mathbf{A d j})\}$ \\
& Weyl & $4 \times\{(N, \bar{N})+(\bar{N}, N)\}$ \\
\hline
\end{tabular}

Table 3: $\quad$ Momentum picture: open string spectrum of $S^{1} / T$ for finite $R_{9}$ 
We see indeed that the massless spectrum is the one of four-dimensional, nonsupersymmetric Yang-Mills with gauge group $U(N) \times U(N)$. The massless spectrum agrees with the open string spectrum of D3- and D3'-branes in the type 0B string. Therefore in the limit $R_{9} \rightarrow 0$ the massive states with masses proportional to $(2 m+1) / R_{9}$ decouple, and we get precise agreement with the type $0 \mathrm{~B}$ string.

The limit $R_{9} \rightarrow \infty$ provides on the other hand an infinite number of new massless momentum states which enhance the gauge symmetry to the group $U(2 N)$ and also restores space-time supersymmetry. So in the large radius limit the spectrum is given by supersymmetric $U(2 N)$ Yang-Mills theory in five space-time dimensions, where 16 supercharges are preserved in the open string theory.

In general starting with $2 N$ wrapped Dp-branes in type $\operatorname{IIA}(\mathrm{B})$ on $S^{1} /(-1)^{F_{s}} S$ one interpolates between the following two decompactification limits:

$R_{9} \rightarrow 0: \quad \mathrm{N} \mathrm{D}(\mathrm{p}-1)$ and $\mathrm{N} \mathrm{D}(\mathrm{p}-1)$ '-branes of type $0 \mathrm{~B}(\mathrm{~A})$ with p-dimensional, nonsupersymmetric $U(N) \times U(N)$ gauge theory.

$R_{9} \rightarrow \infty: 2 \mathrm{~N}$ Dp-branes of type IIA(B) with $(\mathrm{p}+1)$-dimensional, supersymmetric $U(2 N)$ gauge theory.

\subsection{D-branes and open strings in type II on $S^{1} \otimes S^{1} /(-1)^{F_{S}} S$}

So far we have interpolated between supersymmetric and non-supersymmetric gauge theories living in different number of space-time dimensions. Now we will extend the discussion by compactifying the type II string on a two-dimensional compact space given by

$S^{1} \otimes\left(S^{1} /(-1)^{F_{s}} S\right)$ characterized by the two radii $R_{8}$ and $R_{9}$. Using the T-duality on the compact circle in $x_{8}$ we can now smoothly interpolate between D-branes of the same world volume dimensions, and hence also between non-supersymmetric and supersymmetric gauge theories of the same dimensionality.

\section{(i) The winding picture: transversal Dp-branes}

Let us consider $2 \mathrm{~N}$ Dp-branes in type IIA(B) which are transversal to both the $x_{8}$ and the $x_{9}$ direction. The positions of the Dp-branes on the orbifold circle are as discussed in section (2.1). The spectrum of this theory follows without large efforts from the previous discussion. For finite values of the two radii $R_{8}$ and $R_{9}$ we are dealing with a $(\mathrm{p}+1)$ dimensional gauge theory, living on the world volume of the Dp-branes. The massless states are those of maximally supersymmetric $U(N)$ Yang-Mills gauge theory. Of particular interest are the following four possible decompactification limits: 
a) $R_{8} \rightarrow \infty, R_{9} \rightarrow \infty$ : Here we are dealing with Dp-branes of type IIA(B), and the corresponding gauge theory is $(\mathrm{p}+1)$-dimensional, supersymmetric Yang-Mills with $U(N)$ gauge group.

b) $R_{8} \rightarrow 0, R_{9} \rightarrow \infty$ : This limit describes $\mathrm{D}(\mathrm{p}+1)$-branes of type $\operatorname{IIB}(\mathrm{A})$ with $(\mathrm{p}+2)$ dimensional, supersymmetric $U(N)$ gauge theory.

c) $R_{8} \rightarrow \infty, R_{9} \rightarrow 0:$ Now the decompactification limit corresponds to self-dual $\mathrm{D}(\mathrm{p}+1)$ and $\mathrm{D}(\mathrm{p}+1)$ '-branes of type $0 \mathrm{~B}(\mathrm{~A})$ with non-supersymmetric $U(N) \times U(N)$ gauge theory in $\mathrm{p}+2$ dimensions.

d) $R_{8} \rightarrow 0, R_{9} \rightarrow 0$ : Finally we obtain self-dual $\mathrm{D}(\mathrm{p}+2), \mathrm{D}(\mathrm{p}+2)$ '-branes of type $0 \mathrm{~A}(\mathrm{~B})$ with non-supersymmetric $U(N) \times U(N)$ gauge theory in $\mathrm{p}+3$ dimensions.

Therefore, in the winding picture the moduli space of this two-dimensional compactification enables us to interpolate between N D3-branes of type IIB and N self-dual D3, D3'-branes of type 0B. Hence there exists a stringy interpolation mechanism between fourdimensional, non-supersymmetric $U(N) \times U(N)$ gauge theory with 6 adjoint scalars plus $4 \times\{(N, \bar{N})+(\bar{N}, N)\}$ Weyl fermions and four-dimensional $\mathcal{N}=4$ supersymmetric $U(N)$ Yang-Mills theory. The supersymmetric $U(N)$ gauge group is just the diagonal subgroup of the non-supersymmetric $U(N) \times U(N)$ gauge symmetry.

\section{(ii) The momentum picture: wrapped Dp-branes}

In the momentum picture we are considering $2 \mathrm{~N}$ Dp-branes which are wrapped both in the $x_{8}$ and also in the $x_{9}$ directions. For finite values of the radii there is a nonsupersymmetric $U(N) \times U(N)$ gauge theory with massless fields living in p-1 uncompactified space-time dimensions. Again we like to consider the four special decompactification limits:

a) $R_{8} \rightarrow \infty, R_{9} \rightarrow \infty$ : Here we are dealing with Dp-branes of type $\operatorname{IIA}(\mathrm{B})$, and the corresponding gauge theory is $(\mathrm{p}+1)$-dimensional, supersymmetric Yang-Mills with $U(2 N)$ gauge group.

b) $R_{8} \rightarrow 0, R_{9} \rightarrow \infty$ : This limit describes $\mathrm{D}(\mathrm{p}-1)$-branes of type $\operatorname{IIB}(\mathrm{A})$ with pdimensional, supersymmetric $U(2 N)$ gauge theory.

c) $R_{8} \rightarrow \infty, R_{9} \rightarrow 0$ : Now the decompactification limit corresponds to self-dual $\mathrm{D}(\mathrm{p}-1)$ and $\mathrm{D}(\mathrm{p}-1)$ '-branes of type $0 \mathrm{~B}(\mathrm{~A})$ with non-supersymmetric $U(N) \times U(N)$ gauge theory in $\mathrm{p}$ dimensions. 
d) $R_{8} \rightarrow 0, R_{9} \rightarrow 0$ : Finally we obtain self-dual $\mathrm{D}(\mathrm{p}-2), \mathrm{D}(\mathrm{p}-2)$ '-branes of type $0 \mathrm{~A}(\mathrm{~B})$ with non-supersymmetric $U(N) \times U(N)$ gauge theory in p-1 dimensions.

We see that within the momentum picture one can again interpolate between 2N D3branes of type IIB and N self-dual D3, D3'-branes of type 0B. This time it provides an interpolation mechanism between four-dimensional, non-supersymmetric $U(N) \times U(N)$ gauge theory with 6 adjoint scalars plus $4 \times\{(N, \bar{N})+(\bar{N}, N)\}$ Weyl fermions and four-dimensional $\mathcal{N}=4$ supersymmetric $U(2 N)$ Yang-Mills theory. Here the nonsupersymmetric $U(N) \times U(N)$ gauge group is a regular subgroup of the supersymmetric $U(2 N)$ gauge symmetry.

\section{Type 0 compactifications on freely acting orbifolds}

Alternatively we can start also with the type 0A (B) string compactified on $S^{1}$. Modding by $(-1)^{f_{L}}$ leads back to the type IIA (B) superstring theories. It follows that the compactification of type 0A (B) on the freely acting orbifold $S^{1} /(-1)^{f_{L}} S$ possesses the following decompactification limits. For $R_{9} \rightarrow \infty$ one recovers the ten-dimensional nonsupersymmetric type 0A (B) theories and for $R_{9} \rightarrow 0$ the ten-dimensional supersymmetric type IIB (A) theory arises. Observe that now a tachyon develops for large radii $R_{9}>\sqrt{2}$. As was shown in [51 the type 0A (B) orbifold is T-dual to the type IIB (IIA) orbifolds discussed in the previous section, where the relation between the radii is $R_{I I}=2 / R_{0}$. The straightforward T-dual of the type IIB (IIA) over $S^{1} /(-1)^{F_{S}} S$ orbifold is of course type IIA (IIB) over $S^{1} /(-1)^{F_{S}} \tilde{S}$, where $\tilde{S}$ is a shift in the momentum lattice acting as $(-1)^{n}$ on winding modes.

Let us now discuss the properties of the D-branes and the gauge theories in the open string sector within this orbifold compactification of the type 0 string. Since the situation is just T-dual to the orbifold compactification of the type II strings discussed before, we will only summarize the main results. In order to interpolate between type 0 and type II we will only allow for self-dual D-branes in the type 0 orbifolds, i.e. we are placing $\mathrm{N}$ electric Dp and N magnetic Dp'-branes either transversal to the orbifold or wrapped around it.

\section{(i) The winding picture: transversal Dp-branes}

Here we are placing $\mathrm{N}$ electric Dp and $\mathrm{N}$ magnetic Dp'-branes both at $x_{9}=0$ and at $x_{9}=R_{9} / 2$. The action of $(-1)^{f_{L}} S$ on the CP-factors relates for instance a Dp-brane at 
$x_{9}=0$ to a Dp'-brane at $x_{9}=R_{9} / 2$. Using the T-duality relation between the type II and type 0 radii, one obtains exactly the annulus partition function in (2.9). For generic radii the open string sector is given by $(\mathrm{p}+1)$-dimensional, non-supersymmetric $U(N) \times$ $U(N)$ gauge theory. For $R_{9} \rightarrow 0$ additional gauge bosons as well as their superpartners become massless, such that this limit is described by $\mathrm{D}(\mathrm{p}+1)$-branes with associated $\mathcal{N}=4$ supersymmetric $U(2 N)$ gauge theory in $\mathrm{p}+2$ space-time dimensions. On the other hand, for $R_{9} \rightarrow \infty$ we obtain the Dp, Dp'-branes of type 0 , and the gauge theory is nonsupersymmetric $U(N) \times U(N)$ Yang-Mills theory. Of course, we can extend this picture by considering a type 0 compactification on $S^{1} \otimes S^{1} /(-1)^{f_{L}} S$. In this way we get the T-dual picture of the interpolating models discussed in section 2.3.

\section{(ii) The momentum picture: wrapped Dp-branes}

Here the situation is similar to the winding picture in the type II orbifold compactification. We are wrapping $\mathrm{N}$ electric Dp and $\mathrm{N}$ magnetic Dp'-branes around the circle and using the T-duality relation for the radii we obtain the same result as in (2.4). For $R_{9} \rightarrow 0$ the theory becomes $\mathcal{N}=4$ supersymmetric, but now with $U(N)$ gauge group. In contrast, for $R_{9} \rightarrow \infty$ supersymmetry is completely broken but the gauge group is enhanced to $U(N) \times U(N)$.

\section{M-theory embedding}

\subsection{The bulk theory}

So far we have considered embeddings of supersymmetric and non-supersymmetric gauge theories into string theory and by varying some stringy parameters we have seen that one can interpolate between them. Unfortunately, this does not teach us anything new about the dynamics of the non-supersymmetric gauge theories. Therefore, we should try to lift the whole picture to M-theory where at least one of the radii is related to the string coupling constant and therefore to the gauge coupling constant.

Let us briefly review the suggestion of [51] where a freely acting orbifold of 11dimensional M-theory was constructed with the conjecture that the non-supersymmetric type $0 \mathrm{~A}$ string can be viewed as an M-theory compactification on $S^{1} /(-1)^{F_{s}} S$. As a consequence of this conjecture it follows that at small radius $r_{10}$, i.e. at weak string coupling, one recovers the non-supersymmetric type $0 \mathrm{~A}$ string, whereas at large radius $r_{10}$, i.e. at strong string coupling, the maximally supersymmetric M-theory in 11 dimensions emerges. 
Hence the type 0A string should contain fermionic solitons with masses $m_{f}=1 / r_{10}$, and the tachyon of type $0 A$ should become massive at strong coupling. Similarly it was argued in [51] that the ten-dimensional type 0B string can be obtained as the zero volume limit of M-theory on $T^{2} /(-1)^{F_{s}} S$.

So let us consider M-theory compactified on the freely acting orbifold $S^{1} \otimes S^{1} /(-1)^{F_{s}} S$. The corresponding two radii are called $r_{9}$ and $r_{10} \cdot{ }^{6}$ If the $\mathbb{Z}_{2}$ orbifold is in the $x_{9}$ direction then the previous perturbative discussion on the interpolation between supersymmetric and non-supersymmetric theories applies. However if we exchange the role of the two circles such that supersymmetry is broken by the M-theory coordinate $x_{10}$, one has to conclude that the type $0 \mathrm{~A}$ string at weak coupling is given by M-theory on $S^{1} /(-1)^{F_{s}} S$ at zero radius $r_{10}$. Varying the $\mathrm{M}$-theory radius $r_{10}$, i.e. the $0 \mathrm{~A}$ coupling constant $g_{10}^{A}$, one is interpolating between M-theory with 32 supercharges and type $0 \mathrm{~A}$ string with completely broken supersymmetry. To be precise let us recall the well-known relations [55,56] between the string and M-theory parameters. First the relation between the string coupling constant $g_{10}^{A}$ and $r_{10}$ is given by ${ }^{7}$

$$
g_{10}^{A}=\left(\frac{r_{10}}{2}\right)^{3 / 2} .
$$

Next we consider the compactification of M-theory to nine dimensions. The ninedimensional radius of type $0 \mathrm{~A}$ on $S^{1}$ is then related to the M-theory parameters as

$$
R_{9}^{A}=r_{9} \sqrt{\frac{r_{10}}{2}}
$$

whereas the nine-dimensional $0 \mathrm{~A}$ string coupling constant reads

$$
g_{9}^{A}=\frac{g_{10}^{A}}{\sqrt{R_{9}^{A}}}=\left(\frac{r_{10}}{2}\right)^{5 / 4} r_{9}^{-1 / 2}
$$

Using the T-duality between $0 \mathrm{~A}$ and $\mathrm{OB}$ in nine dimensions we can express the $0 \mathrm{~B}$ parameters in the following way:

$$
R_{9}^{B}=\frac{1}{R_{9}^{A}}=\frac{\sqrt{2}}{r_{9} \sqrt{r_{10}}}, \quad g_{9}^{B}=g_{9}^{A}=\left(\frac{r_{10}}{2}\right)^{5 / 4} r_{9}^{-1 / 2}, \quad g_{10}^{B}=g_{9}^{B} \sqrt{R_{9}^{B}}=\frac{r_{10}}{2 r_{9}} .
$$

${ }^{6}$ Radii measured in units of the 11-dimensional Planck length $L_{11}$ are denoted by small letters. On the other hand, radii measured in units of the string scale $\sqrt{\alpha^{\prime}}$ are denoted as before by capital letters.

7 The relation between the type 0 parameters and M-theory parameters are as in the type II case except some additional factors of 2 due to the orbifoldization. 
Using these relations we are interested in the following three type IIB (0B) limits.

a) $r_{9} \rightarrow 0, r_{10} \rightarrow 0$ with $R_{9}^{B} \rightarrow \infty$ : Here we obtain the type $0 \mathrm{~B}$ string at finite or zero string coupling $g_{10}^{B}=r_{10} / 2 r_{9}$.

b) $r_{9} \rightarrow 0, r_{10} \rightarrow \infty$ with $R_{9}^{B} \rightarrow \infty$ : This limit describes the strongly coupled type IIB string with $g_{10}^{B} \rightarrow \infty$.

c) $r_{9} \rightarrow \infty, r_{10} \rightarrow 0$ with $R_{9}^{B} \rightarrow \infty$ : Now one is dealing with the weakly coupled type 0B string with $g_{10}^{B} \rightarrow 0$.

Let us now discuss how M-theory branes and the corresponding gauge theories can be incorporated into this picture.

\subsection{M2-branes}

The membrane solution (M2-brane) of 11-dimensional supergravity plays a very important role in the relation between string theory and M-theory. Upon circle compactification from 11 to 10 dimensions M2-branes with world volumes transversal to $x_{10}$ can be identified with the IIA D2-branes. On the other hand, being wrapped around the eleventh dimension the M2-brane provides the fundamental string of the IIA superstring. Let us now discuss the fate of the M2-branes under the M-theory compactification on $S^{1} \times S^{1} /(-1)^{F_{s}} S$, where the orbifold lies in the $x_{10}$ direction. As a first set of membranes we introduce $2 \mathrm{~N} \mathrm{M}$-branes which are completely unwrapped, so that their worldvolumes are transversal to the compact two-dimensional orbifold. Therefore this choice corresponds to the winding picture discussed before. Of course we have to place N M2-branes at opposite positions on the circle. In order to obtain gauge theories the M2-branes are intersected by another set of M2-branes which are wrapped around the orbi-circle in $x_{10}$. Hence from the string point of view these wrapped membranes correspond to the open strings which are responsible for the gauge symmetry degrees of freedom.

In order to see what the various limits of M-theory parameters mean for the corresponding gauge theories we need the relations between string theory and M-theory parameters, where we measure all length scales in units of the 11-dimensional Planck length $L_{11}$, which now appears explicitly in all formulas, i.e. $r_{9}$ and $r_{10}$ are now dimensionful(!) quantities. Focussing on type B quantities we obtain:

$$
R_{9}^{B}=\frac{2 L_{11}^{3}}{r_{9} r_{10}}, \quad g_{10}^{B}=\frac{r_{10}}{2 r_{9}}
$$


Note that switching from $R_{9}^{B}$ measured in string units to the radius measured in M-theory units the following relation between the fundamental string scale $\alpha^{\prime}$ and $L_{11}$ is required:

$$
T_{F}=\frac{1}{\alpha^{\prime}}=\frac{r_{10}}{2 L_{11}^{3}}
$$

The ten-dimensional gravitational coupling constant is given by

$$
\kappa_{10}^{2}=\frac{L_{11}^{9}}{r_{10}} .
$$

In addition we also need the expression for the tension of the solitonic D1-strings and D3-branes:

$$
T_{D 1}=\frac{T_{F}}{g_{10}^{B}}=\frac{r_{9}}{L_{11}^{3}}, \quad T_{D 3}=\frac{T_{F}^{2}}{g_{10}^{B}}=\frac{r_{9} r_{10}}{2 L_{11}^{6}} .
$$

In the following we like to consider the gauge theories in the limit $R_{9}^{B} \rightarrow \infty$, i.e. in the type B limit. Moreover we like to study the behavior of the gauge theories in the limit where the 11-dimensional Planck length is small, i.e. $L_{11}=\rho \rightarrow 0$.

a) $r_{9}=\rho^{a} \rightarrow 0(a>0), r_{10}=\rho^{b} \rightarrow 0(b>0):$

In order that $R_{9}^{B} \rightarrow \infty$ we have to require that $a+b>3$. Then this limit describes the nonsupersymmetric type 0B string. It contains $\mathrm{N}$ self-dual D3-branes. In order that the gauge theory modes completely decouple from the perturbative as well as all non-perturbative stringy modes we demand in addition that $T_{F} \rightarrow \infty$ and $T_{D 1} \rightarrow \infty$. This provides a further restriction on how $r_{9}$ and $r_{10}$ go to zero, namely $a<3$ and also $b<3$. Choosing the parameters in this way we have a non-supersymmetric $U(N) \times U(N)$ gauge theory with 6 adjoint scalars and $4+4$ bifundamental fermions in four dimensions. The gauge theory is weakly coupled, $g_{Y M}=\sqrt{g_{10}^{B}} \rightarrow 0$, if $b>a$. Moreover this non-supersymmetric gauge theory is expected to possess an S-duality symmetry $g_{Y M} \rightarrow 1 / g_{Y M}$, which is realized by the exchange of $r_{9}$ and $r_{10}$. Thus the strongly coupled non-supersymmetric $U(N) \times U(N)$ gauge theory is obtained choosing $a>b$. Of course, one has to be very careful with such duality statements in the non-supersymmetric case, as the lack of supersymmetry does not allow us to find more supporting evidence for such a conjecture.

b) $r_{9}=\rho^{a} \rightarrow 0(a>0), r_{10}=\rho^{-b} \rightarrow$ const. or $\infty(b \geq 0)$ :

Choosing the two radii in this way we run towards supersymmetry restoration; supersymmetry is completely restored for $r_{10} \rightarrow \infty$. Again keeping $R_{9}^{B}$ large we need $a>3+b$, i.e. 
$a>3$. Then in the supersymmetric limit the N M2-branes can be viewed as type IIB D3branes with open strings ending on them. The open strings lead to $\mathcal{N}=4$ supersymmetric $U(N)$ gauge theory at very strong Yang-Mills gauge coupling $g_{Y M}=\sqrt{g_{10}^{B}} \rightarrow \infty$. However now the stringy modes do not decouple anymore. Although the fundamental string tension $T_{F}$ is still very large, the D1 string tension $T_{D 1}$ goes to zero, since $a>3$. This means that we deal not only with strongly coupled $\mathcal{N}=4$ supersymmetric $U(N)$ Yang-Mills, but also all D-stringy modes are present and do not decouple. We call this theory $\mathcal{N}=4 \mathrm{MSYM}$.

c) $r_{9}=\rho^{-a} \rightarrow$ const. or $\infty(a \geq 0), r_{10}=\rho^{b} \rightarrow 0(b>0)$ :

In this limit supersymmetry is completely broken. Asking for $R_{9}^{B} \rightarrow \infty$ it implies $b>3+a$, i.e. $b>3$. Then the N M2-branes can be viewed as self-dual type 0B D3-branes with non-supersymmetric $U(N) \times U(N)$ gauge theory at very weak Yang-Mill gauge coupling

$g_{Y M}=\sqrt{g_{10}^{B}} \rightarrow 0$. Now the elementary stringy modes do not decouple since $T_{F} \rightarrow 0$ whereas $T_{D 1} \rightarrow \infty$. This means that we deal not only with classical non-supersymmetric $U(N) \times U(N)$ Yang-Mills theory, but also all elementary stringy modes are present and do not decouple. Let us call this theory $\mathcal{N}=0$ MYM theory. Under exchange of the two compact radii this case is mapped to the limit described in b.), implying some strong-weak duality between these two supersymmetric resp. non-supersymmetric gauge theories.

In all three type IIB (0B) limits the ten-dimensional gravitational coupling vanishes and the tension of the D3-branes becomes infinite, so that gravity and massive modes on the D3-branes decouple properly. If we take the M-theory discussion seriously, it tells us that the non-supersymmetric $U(N) \times U(N)$ gauge theory can be continued at very weak and very strong coupling to two new branches of gauge like theories, where not all the stringy modes decouple from the dynamics. On one of these two branches the model can be deformed continuously to a supersymmetric model. Moreover, analogously to the bulk theory one might expect a strong-weak duality for the non-supersymmetric $U(N) \times U(N)$ gauge theory for finite gauge coupling. In the large $\mathrm{N}$ limit this might really be true, as the theory is conformal and the gauge coupling is a free parameter.

\subsection{M5-branes}

The solitonic solution dual to the membranes in 11-dimensional supergravity are given by the M-theory 5-branes (M5-branes). We will consider 2N M5-branes which are wrapped around both directions of the compact space $S^{1} \otimes S^{1} /(-1)^{F_{s}} S$. Hence from the string 
point of view we are in the momentum picture. Therefore in nine dimensions the M5branes correspond to $2 \mathrm{~N}$ D3-branes. These $2 \mathrm{~N}$ M5-branes are intersected by M2-branes which correspond to the open string sector in string theory. In complete analogy to the unwrapped case, in the three type IIB (0B) limits one obtains the following spectra.

a) $r_{9}=\rho^{a} \rightarrow 0(a>0), r_{10}=\rho^{b} \rightarrow 0(b>0)$ : We obtain N self-dual D3 plus D3'-branes of finitely coupled type 0B. Now we have non-supersymmetric $U(N) \times U(N)$ gauge theory with the usual matter content.

b) $r_{9}=\rho^{a} \rightarrow 0(a>0), r_{10}=\rho^{-b} \rightarrow$ const. or $\infty(b \geq 0)$ : Here we are dealing with the strongly coupled type IIB. After T-duality in the $x_{9}$ direction the $2 \mathrm{~N}$ M5-branes can be viewed as $2 \mathrm{~N}$ D3-branes with open strings ending on them. The corresponding gauge theory in the momentum picture is given by four-dimensional, $\mathcal{N}=4$ supersymmetric $U(2 N)$ gauge theory at very strong Yang-Mill gauge coupling. However, not all D1-stringy modes decouple and one does again get $\mathcal{N}=4$ MSYM with gauge group $U(2 N)$.

c) $r_{9}=\rho^{-a} \rightarrow$ const. or $\infty(a \geq 0), r_{10}=\rho^{b} \rightarrow 0(b>0)$ :

Now one is dealing with N D3 plus N D3'-branes of weakly coupled type 0B string theory. The corresponding gauge theory is weakly coupled $\mathcal{N}=0 \mathrm{MYM}$ with gauge group $U(N) \times U(N)$.

In the M5-brane scenario we get two new branches at very small and very large coupling, on which the supersymmetric $U(2 N)$ and the non-supersymmetric $U(N) \times U(N)$ gauge theory do still couple to some stringy modes.

\section{Conclusions}

In this paper we have shown that D-branes in freely acting orbifolds of type II and type 0 string constructions allow for a continuous interpolation between $\mathcal{N}=4$ supersymmetric gauge theories with $G=U(N)$ or $G=U(2 N)$ gauge symmetry and a non-supersymmetric gauge theory with gauge group $G=U(N) \times U(N), 6$ adjoint scalars in each gauge group factor plus 4 Weyl fermions in the representations $(N, \bar{N})+(\bar{N}, N)$. The interpolation mechanism is of stringy nature and can be realized varying the radii of the compact orbifold space. Therefore the coupling of the open string degrees of freedom to the modes of the closed string compactification are crucial for the understanding of this mechanism.

We have also discussed how this scenario can be lifted to M-theory. As a result of this discussion a non-supersymmetric gauge theory at weak coupling can be continuously 
connected to a supersymmetric gauge theory like branch at strong coupling. Another conclusion from this investigation was that the non-supersymmetric gauge theory itself might have a strong-weak coupling duality. Let us emphasize, that duality conjectures for non-supersymmetric models are on less solid ground as compared to the supersymmetric case. Therefore, the M-theory results should be considered with some care.

It is clear that the discussion in this paper can be straightforwardly generalized putting branes on transversal singularities or discussing Hanany-Witten type of brane constructions. Consider for example the case of D3-branes probing a transversal, non-compact $\mathbb{Z}_{K}$ orbifold. Via T-duality this is equivalent to $K$ NS 5 -branes intersected by D4-branes [57,58. For the type II case, supersymmetry is broken to $\mathcal{N}=2$ and the gauge group is now given by $G=U(N)^{K}$. In the corresponding type 0 string the same construction leads to a non-supersymmetric gauge theory with gauge group $[U(N) \times U(N)]^{K}$ plus certain matter fields. Again the large $\mathrm{N} \beta$-function is the same as in the $\mathcal{N}=2$, type II parent model. As before the freely acting orbifold construction implies that one can interpolate between these two $\mathcal{N}=2$ and $\mathcal{N}=0$ gauge theories. For orbifolds or conifolds which break the amount of supersymmetry on the brane down to $\mathcal{N}=1$, an interpolating model can be build in an analogous way.

\section{Acknowledgements}

We thank C. Angelantonj, M. Green, A. Karch, I. Klebanov and A. Sagnotti for useful discussions. The work is partially supported by the European Commission TMR program under the contract ERBFMRXCT960090, in which the Humboldt-University at Berlin and the Ecole Normale Superieur in Paris are associated. 


\section{References}

[1] J. M. Maldacena, The Large N Limit of Superconformal Field Theories and Supergravity, Adv.Theor.Math.Phys. 2 (1998) 231, hep-th/9711200.

[2] S.S. Gubser, I.R. Klebanov and A.M. Polyakov, Gauge Theory Correlators from Noncritical String Theory, Phys. Lett. B428 (1998) 105, hep-th/9802109.

[3] E. Witten, Anti-de Sitter Space and Holography, Adv. Theor. Math. Phys. 2 (1998) 253, hep-th/9802150.

[4] E. Witten, Anti-de Sitter Space, Thermal Phase Transition and Confinement in Gauge Theories, Adv, Theor, Math. Phys. 505 (1998) 505, hep-th/9803131.

[5] I.R. Klebanov and A.A. Tseytlin, D-Branes and Dual Gauge theories in Type 0 Strings, Nucl.Phys. B546 (1999) 155, hep-th/9811035.

[6] I.R. Klebanov and A.A. Tseytlin, A Non-supersymmetric Large N CFT from Type 0 String Theory, JHEP 9903 (1999) 015, hep-th/9901101.

[7] I.R. Klebanov and A.A. Tseytlin, Asymptotic Freedom and Infrared Behavior in the Type 0 String Approach to Gauge Theory, Nucl. Phys. B547 (1999) 143, hepth/9812089

[8] A.M. Polyakov, The Wall of the Cave, Int.J.Mod.Phys. A14 (1999) 645, hepth/9809057.

[9] L. Dixon and J. Harvey, String Theories in Ten Dimensions Without Space-Time Supersymmetry, Nucl. Phys. B274 (1986) 93.

[10] N. Seiberg and E. Witten, Spin Structures in String Theory, Nucl. Phys. B276 (1986) 272.

[11] N. Nekrasov and S.L. Shatashvili, On Non-Supersymmetric CFT in Four Dimensions, hep-th/9902110.

[12] S. Kachru, J. Kumar, E. Silverstein, Vacuum Energy Cancellation in a Nonsupersymmetric String, Phys. Rev. D59 (1999) 106004, hep-th/9807076; S. Kachru and E. Silverstein, Self-Dual Nonsupersymmetric Type II String Compactifications, JHEP 9811 (1998) 001, hep-th/9808056; S. Kachru and E. Silverstein, On Vanishing Two Loop Cosmological Constant in Nonsupersymmetric Strings, JHEP 9901 (1999) 004, hep-th/9810129.

[13] J. A. Harvey, String Duality and Non-supersymmetric Strings, Phys.Rev. D59 (1999) 026002, hep-th/9807213.

[14] G. Shiu and S.-H.H Tye, Bose-Fermi Degeneracy and Duality in Non-Supersymmetric Strings, Nucl.Phys. B542 (1999) 45, hep-th/9808095.

[15] R. Blumenhagen, L. Görlich, Orientifolds of Non-Supersymmetric Asymmetric Orbifolds, Nucl.Phys. B551 (1999) 601, hep-th/9812158.

[16] C. Angelantonj, I. Antoniadis, K. Förger, Non-Supersymmetric Type I Strings with Zero Vacuum Energy, hep-th/9904092. 
[17] A. Sagnotti, M. Bianchi, On the Systematics of Open String Theories, Phys. Lett. B247 (1990) 517

[18] A. Sagnotti, Some Properties of Open String Theories, hep-th/95090808;

A. Sagnotti, Surprises in Open String Perturbation Theory, hep-th/9702093.

[19] O. Bergman and M.R. Gaberdiel, A Non-Supersymmetric Open String Theory and S-Duality, Nucl.Phys. B499 (1997) 183, hep-th/9701137.

[20] C. Angelantonj, Non-Tachyonic Open Descendants of the OB String Theory, Phys.Lett. B444 (1998) 309, hep-th/9810214.

[21] R. Blumenhagen, A. Font and D. Lüst, Tachyon-free Orientifolds of Type OB Strings in Various Dimensions, hep-th/9904069.

[22] R. Blumenhagen and A. Kumar, A Note on Orientifolds and Dualities of Type OB String Theory, hep-th/9906234.

[23] K. Förger, On Non-tachyonic $Z_{N} \times Z_{M}$ Orientifolds of Type 0B String Theory, hepth/9909010.

[24] M.R. Douglas and G. Moore, D-branes, Quivers and ALE Instantons, hep-th/9603167.

[25] S. Kachru and E. Silverstein, 4d Conformal Field Theories and Strings on Orbifolds, Phys. Rev. Lett. 80 (1998) 4855, hep-th/9802183.

[26] A. Lawrence, N. Nekrasov and C. Vafa, On Conformal Field Theories in Four Dimensions, Nucl. Phys. B533 (1998) 199, hep-th/9803076.

[27] I.R. Klebanov and E. Witten, Superconformal field theory on three-branes at a CalabiYau singularity, Nucl. Phys. B536 (1998) 199, hep-th/9807080.

[28] A. Hanany and E. Witten, TypeIIB Superstrings, BPS monopoles and Three Dimensional Gauge Dynamics, Nucl. Phys. B492 (1997) 152, hep-th/9611230.

[29] M. Alishahiha, A. Brandhuber and Y. Oz, Branes at Singularities in Type 0 String Theory, JHEP 9905 (1999) 024, hep-th/9903186.

[30] M. Billo, B. Craps and F. Roose, On D-branes in Type O String Theory, hepth/9902196.

[31] A. Armoni and B. Kol, Non-Supersymmetric Large $N$ Gauge Theories from Type 0 Brane Configurations, hep-th/9906081.

[32] R. Blumenhagen, A. Font and D. Lüst, Non-supersymmetric Gauge Theories from D-branes in Type 0 String Theory, hep-th/9906101.

[33] I.R. Klebanov, N.A. Nekrasov and S.L. Shatashvili, An Orbifold of Type OB Strings and Non-supersymmetric Gauge Theories, hep-th/9909109.

[34] J. Scherk and J.H. Schwarz, Spontaneous Breaking of Supersymmetry through Dimensional Reduction, Phys. Lett. 82B (1979) 60.

[35] C. Kounnas and M. Porrati, Spontaneous Supersymmetry Breaking in String Theory Nucl. Phys. B310 (198) 355.

[36] S. Ferrara, C. Kounnas, M. Porrati and F. Zwirner, Superstrings with Spontaneously Broken Supersymmetry and their Effective Theories, Nucl. Phys. B318 (1989) 76. 
[37] C. Kounnas and B. Rostand, Coordinate Dependent Compactification and Discrete Symmetries, Nucl. Phys. B341 (1990) 641.

[38] C. Kounnas, BPS States in Superstrings with Spontaneously Broken SUSY, Nucl. Phys. Proc.Suppl.B58 (1997) 57.

[39] E. Kiritsis and C. Kounnas, Perturbative and Nonperturbative Supersymmetry Breaking: $N=4 \rightarrow N=2 \rightarrow N=1$ Nucl. Phys. B503 (1997) 117.

[40] B. Sathiapalan, Vortices on the String World Sheet and Constraints on Toroidal Compactification, Phys. Rev. D35 (1987) 3277.

[41] I. Kogan, Vortices on the World Sheet and String's Critical Dynamics, JETP Lett. 45 (1987) 709.

[42] J.J. Attick and E. Witten, The Hagedorn Transition and the Number of Degrees of Freedom of String Theory, Nucl. Phys. B310 (1988) 291.

[43] I. Antoniadis and C. Kounnas, Supersymmetric Phase Transition at High Temperature, Phys. Lett. B261 (1991) 369.

[44] I. Antoniadis, J.P. Derendinger and C. Kounnas, Nonperturbative Temperature Instabilities in N=4 Strings Nucl. Phys. B551 (1999) 41.

[45] I. Antoniadis, J.P. Derendinger and C. Kounnas, Nonperturbative Supersymmetry Breaking and Finite Temperature Instabilities in N=4 Superstrings, hep-th/9908137.

[46] J.D. Blum and K.R. Dienes, Duality without Supersymmetry: The Case of the SO(16)×SO(16) String, Phys.Lett. B414 (1997) 260, hep-th/9707148; Strong/Weak Coupling Duality Relations for Non-Supersymmetric String Theories, Nucl.Phys. B516 (1998) 83, hep-th/9707160.

[47] I. Antoniadis, E. Dudas and A. Sagnotti, Brane Supersymmetry Breaking, hepth/9908023.

[48] I. Antoniadis, G. D'Appollonio, E. Dudas and A. Sagnotti, Partial Breaking of Supersymmetry, Open Strings and M-theory, Nucl. Phys. B553 (1999) 133, hep-th/9812118.

[49] I. Antoniadis, E. Dudas and A. Sagnotti, Supersymmetry Breaking, Open Strings and M-theory, Nucl. Phys. B544 (1999) 469, hep-th/9807011.

[50] I. Antoniadis and M. Quiros, Supersymmetry Breaking in M-theory and Gaugino Condensation, Nucl. Phys. B505 (1997) 109, hep-th/9705037; On the M-theory Description of Gaugino Condensation, Phys. Lett. B416 (1998) 327, hep-th/9707208.

[51] O. Bergman and M.R. Gaberdiel, Dualities of Type 0 Strings, hep-th/9906055.

[52] M. Dine, P. Huet and N. Seiberg, Large and Small Radius in String Theory, Nucl. Phys. B322 (1989) 301.

[53] J. Dai, R.G. Leigh and J. Polchinski, New Connections between String Theories, Mod. Phys. Lett. A4 (1989) 2073.

[54] M. Abou-Zeid, B. de Wit, D. Lüst and H. Nicolai, Space-time Supersymmetry, IIA/B Duality and M-Theory, hep-th/9908169. 
[55] E. Witten, String Theory Dynamics in Various Dimensions, Nucl. Phys. B443 (1995) 85, hep-th/9503124.

[56] J.H. Schwarz, The Power of M Theory, Phys. Lett. B367 (1996) 97, hep-th/9510086; J.H. Schwarz, Lectures on Superstring and M Theory Dualities, Nucl. Phys. Proc. Suppl. B55 (1997) 1, hep-th/9607201.

[57] A. Karch, D. Lüst and D. Smith, Equivalence of Geometric Engineering and HananyWitten via Fractional Branes, Nucl. Phys. B533 (1998) 348, hep-th/9803232.

[58] B. Andreas, G. Curio and D. Lüst, The Neveu-Schwarz Five-brane and its Dual Geometries, JHEP 9810 (1998) 022, hep-th/9807008. 